\title{
Avaliação da dor em pacientes com diagnóstico de câncer de colo do útero em Sergipe
}

\author{
Pain assessment in patients diagnosed with cervical cancer in \\ Evaluación del dolor en pacientes diagnosticados con cáncer cervical en Sergipe
}

Delza Correia Lima ${ }^{1 *}$, Maria Nathália Prado Simões Mendonça1, Ayla Gabriella Silva Ribeiro', Luísa Teixeira Silveira ${ }^{1}$, Tiago Almeida Costa $^{1}$, Marina de Pádua Nogueira Menezes ${ }^{2}$, Bruna Nogueira Viana ${ }^{3}$.

\section{RESUMO}

Objetivo: Avaliação da dor em pacientes com câncer de colo uterino atendidos no Hospital de Urgência de Sergipe (HUSE), tanto do ponto de vista qualitativo quanto quantitativo. Métodos: Estudo quantitativo, descritivo, tipo exploratório, de corte transversal, realizado no ambulatório de Oncologia do HUSE, no município de Aracaju, entre 2010 e 2014. A pesquisa foi realizada em 53 pacientes diagnosticadas e tratadas com câncer de colo de útero e a coleta de dados foi realizada por meio de questionários, como o de Mcgill e a Escala Visual Analógica. Resultados: A queixa álgica foi um fenômeno relatado por 34 pacientes (64,1\%), seja no diagnóstico, no tratamento, na reabilitação ou em mais de uma dessas etapas. Quanto à intensidade da dor, 22 entrevistadas $(41,5 \%)$ apresentaram dor intensa e $19(35,8 \%)$ não apresentaram dor. Ao analisar os descritores da dor, observou-se que dentre as 34 pacientes que referiam a queixa álgica, a categoria sensorial foi preponderante, seguida pela afetiva. O descritor sensitivo mais utilizado foi dor fina com 10,6\%, já no caráter afetivo foi dor enjoada com 22,9\%. Conclusão: A dor intensa esteve presente na maioria das pacientes em algum estágio da doença e o caráter sensorial da queixa álgica foi predominante no estudo.

Palavras-chave: Dor, Avaliação, Câncer de colo uterino.

\begin{abstract}
Objective: Evaluation of pain in patients with cervical cancer treated at Sergipe Emergency Hospital (HUSE), both from a qualitative and quantitative point of view. Methods: Quantitative, descriptive, exploratory, crosssectional study, carried out at the HUSE Oncology Outpatient Clinic, in the municipality of Aracaju, between 2010 and 2014. The research was conducted on 53 patients diagnosed and treated with cervical cancer and data collection was done through questionnaires, such as Mcgill's and the Visual Analog Scale. Results: The pain was a phenomenon reported by 34 patients $(64.1 \%)$, either in diagnosis, treatment, rehabilitation or in more than one of these stages. As for pain intensity, 22 interviewees (41.5\%) presented intense pain and 19 (35.8\%) presented no pain. When analyzing the pain descriptors, it was observed that among the 34 patients who reported the pain, the sensory category was preponderant, followed by the affective one. The most used sensitive descriptor was fine pain with $10.6 \%$, but in the affective character it was nauseated pain with $22.9 \%$. Conclusion: Intense pain was present in most patients at some stage of the disease and the sensory character of the complaint was predominant in the study.
\end{abstract}

Keywords: Pain, Assessment, Cervical cancer.

\section{RESUMEN}

Objetivo: Evaluar el dolor en pacientes con cáncer de cuello uterino atendidas en el Hospital de Emergencia de Sergipe (HUSE), tanto cualitativa como cuantitativamente. Métodos: Estudio cuantitativo, descriptivo, exploratorio, transversal, realizado en el Ambulatorio de Oncología del HUSE, la ciudad de Aracaju, entre 2010 y 2014. La investigación se realizó en 53 pacientes diagnosticadas y tratadas con cáncer de cuello uterino y la recogida de datos se hizo mediante cuestionarios como el Mcgill y la Escala Visual Analógica.

\footnotetext{
1 Universidade Tiradentes (UNIT), Aracaju - SE. *E-mail: delzalima1999@hotmail.com

2 Universidade Federal de Sergipe (UFS), Aracaju - SE.

${ }^{3}$ Centro Universitário Uninovafapi (UNINOVAFAPI), Teresina - PI.
} 
Resultados: La queja fue un fenómeno reportado por 34 pacientes $(64,1 \%)$, tanto en el diagnóstico, el tratamiento, la rehabilitación o más de una de estas etapas. En cuanto a la intensidad del dolor, 22 (41,5\%) presentaron dolor intenso y 19 (35,8\%) no presentaron dolor. Al analizar los descriptores del dolor, se observó que entre los 34 pacientes que refirieron la queja de dolor, predominaba la categoría sensorial, seguida de la afectiva. El descriptor sensible más utilizado fue el dolor fino, con 10,6\% pero el dolor afectivo fueron las náuseas, con $22,9 \%$. Conclusión: El dolor intenso estuvo presente en la mayoría de los pacientes en alguna etapa de la enfermedad y el carácter sensorial de la queja fue predominante en el estudio.

Palabras clave: Dolor, Evaluación, Cáncer cervical.

\section{INTRODUÇÃO}

A dor é uma experiência sensorial e emocional desagradável, associada à lesão tissular real, potencial ou descrita em termos de tal lesão. Esse sintoma ainda é muito negligenciado e mal conduzido por muitos médicos. Por isso, devemos fazer uma avaliação correta da dor para verificar a influência desse sintoma na qualidade de vida do indivíduo e a eficácia do tratamento. O decágono da dor engloba início, local, irradiação, periodicidade, tipo, duração, fatores desencadeantes, fatores de melhora, intensidade, alterações associadas (ARAUJO L e ROMERO B, 2015).

Diante da dificuldade de diagnóstico e mensuração da dor, a Organização Mundial da Saúde (OMS) e a Sociedade Internacional de Estudo da Dor (IASP) criaram escalas, como a Escala Visual Analógica (EVA), que serviu para uniformização internacional e transformação de um sintoma subjetivo em objetivo, facilitando o tratamento (BRASIL, 2001).

A Escala Visual Analógica (EVA) é uma linha horizontal de 10 centímetros, em linha reta, com pontos de ancoragem de nenhuma dor (escore 0 ) e dor insuportável (escore 10). Outra escala mais complexa que a EVA, é a utilizada pelo Questionário de McGill, que permite a avaliação quantitativa e qualitativa da dor, quanto ao seu aspecto sensorial, afetivo e avaliativo (RUELA L, et al., 2017).

O questionário de dor McGill é organizado com 78 descritores distribuídos em 20 grupos de caracterização da dor. Cada grupo pode apresentar de 3 a 5 descritores onde apenas um, o mais adequado para descrição da dor, será assinalado em cada grupo. Os 20 grupos são categorizados em 4 grandes grupos: Sensorial, Afetivo, Avaliativo e Miscelânea. Esta escala apresenta índices de validade e confiabilidade estabelecidos e poder discriminativo entre os diversos componentes da dor (RUELA L, et al., 2017).

O câncer é hoje a segunda causa de mortalidade no Brasil, logo atrás das doenças cardiovasculares, sendo importante o seu controle e prevenção (OLIVEIRA S, et al; 2020). Segundo o Instituto Nacional do Câncer (INCA), foram identificados 576 mil casos novos de câncer no país entre 2014 e 2015. O câncer resulta de um crescimento desordenado de células que se multiplicam rapidamente, formando tumores que podem invadir outros tecidos e órgãos por disseminação direta e/ou pelas vias linfáticas e sanguíneas (GRANER K, et al; 2010).

A prevalência da dor aumenta com a progressão da enfermidade. Cerca de $75 \%$ dos pacientes com câncer avançado sente dor, sendo esse sintoma descrito como moderado (50\%) ou insuportável (30\%). O Ministério da Saúde (MS) considera que a dor causada pelo próprio câncer é responsável por 46 a $92 \%$ dos casos álgicos; a dor relacionada ao câncer, 12 a 29\%; a dor associada ao tratamento antitumoral, 5 a 20\%; dor relacionada a desordens concomitantes, 8 a 22\% (FACINA T, 2014).

A dor oncológica é descrita como "dor total", visto que envolve a interação das reações nociceptivas, emocionais, sociais e espirituais (ARAUJO L e ROMERO B, 2015). O conceito de dor total engloba: aspectos físicos (danos teciduais, progressão da doença e/ou reação à radioterapia); aspectos psicológicos (afeto, mudança de humor, apatia, disposição geral); aspectos sociais (isolamento social, desmotivação geral e convivência prejudicada com a família e outras pessoas relevantes); aspectos espirituais (variações na relação dos indivíduos com suas crenças, princípios e valores, desesperança, sentimentos de desamparo, questionamentos quanto à fé e ao sentido da vida) (GRANER K, et al., 2010). 
A fisiologia da dor oncológica envolve a dor nociceptiva ou a dor neuropática. A dor nociceptiva resulta da liberação pelo tumor de mediadores que sensibilizam e ativam os nociceptores - sejam eles mecânicos, térmicos, polimodais ou silenciosos - aferentes primários, localizados nas fibras nervosas dos tecidos cutâneos ou profundos (SCHMIDT B, 2014).

A dor neuropática depende da indução na reorganização neuroquímica da medula espinhal, o que contribui para a sua atividade espontânea e para o reforço da sua capacidade de resposta (SCHMIDT B, 2014). Essa dor é descrita como uma dor crônica, cujo mecanismo álgico ocorre em algum lugar das vias nociceptivas, não havendo a estimulação dos nociceptores. A dor nociceptiva é subdividida em dor somática e dor visceral. Esta resulta da ativação de nociceptores das vísceras, tecido abdominal ou torácico, é uma dor mal localizada e profunda (sensação de aperto); àquela resulta da estimulação de fibras nervosas aferentes por lesões traumáticas, é mais localizada, pode ser descrita como uma dor desde aguda e intensa até imprecisa e dolorida (ARAUJO L e ROMERO B, 2015).

A dor associada à quimioterapia é resultado da passagem dos antineoplásicos pelos vasos sanguíneos; já na radioterapia, ocorre pela ablação térmica no local do tumor; e nas cirurgias, pode ser por uma lesão nervosa (THOMAZ A, 2010).

A dor no câncer de colo uterino surge nos estágios avançados da doença, assim como nos diferentes tipos de câncer (CASARIN M e PICCOLI J, 2011). Esse tipo de câncer tem uma evolução lenta, sendo assintomático na fase inicial e evoluindo com sinais e sintomas de forma tardia, o que leva as mulheres a procurarem o médico quando já estão em fase avançada diminuindo as chances de um bom prognóstico (CARVALHO K, et al., 2019).

Essa doença apresenta características clínicas variáveis, como quadros de corrimento vaginal persistente (com odor fétido) e de sangramento vaginal anormal. Este sintoma desenvolve-se decorrente de um aumento no fluxo menstrual, de um sangramento vaginal intermitente, intermenstrual, pós-menopausa ou pós-coito (PIMENTEL A, et al; 2011). Em estágios mais avançados pode apresentar também dor abdominal com sintomas relacionados ao aparelho urinário ou intestinal (GREEN MC, et al.,2020).

Cerca de 311 mil mulheres morrem de câncer do colo do útero todos os anos, sendo que mais de $85 \%$ desses óbitos ocorrem nos países subdesenvolvidos (INCA, 2020). O câncer de colo do útero (CCU) possui aproximadamente 570 mil novos casos por ano no mundo, é o quarto tipo de câncer mais comum e quarta causa de morte por câncer em mulheres (INCA, 2020). Outro estudo, afirma que o câncer cervical é o terceiro câncer mais incidente nas mulheres e a quarta causa de óbitos no mundo (BICCA G, et al., 2013).

O câncer do colo uterino é raro em mulheres até 30 anos e sua incidência aumenta progressivamente até ter seu pico na faixa etária de 45 a 50 anos. Esse tipo de neoplasia se destaca como o primeiro mais incidente na região Norte (26,4 casos por 100.000 mulheres); o segundo mais incidente nas regiões Nordeste (16,1/100 mil) e Centro-Oeste (12,5/100 mil); a quarta na região Sul (12,6/100 mil); e a quinta na região Sudeste (8,6/100 mil). Em relação à mortalidade, a região Norte também ocupa o primeiro lugar (12,58 mortes por 100.000 mulheres); em seguida estão as regiões Nordeste (6,66/100 mil), Centro-Oeste (6,32/100 mil), Sul (4,99/100 mil) e Sudeste (3,71/100 mil) (INCA, 2019).

O câncer cervical ou de colo uterino é causado pela infecção persistente por um ou mais tipos oncogênicos do papilomavírus humano (HPV) (NUNES PL, et al., 2020). Este pode ser potencializado por fatores de risco, como o tabagismo, fatores genéticos (como o polimorfismo da proteína p53), imunodepressão, etilismo, multiparidade, baixas condições sócio-econômicas, higiene íntima inadequada, início precoce da atividade sexual, uso prolongado de contraceptivos orais, multiplicidade de parceiros, idade precoce do primeiro parto (SOUSA AC, et al., 2017). O desenvolvimento natural de um câncer invasivo pode ser curto, em torno de dez anos, embora possa desenvolver em cerca de 20 anos (CARVALHO K, et al., 2019).

Os tipos de HPV envolvidos com o câncer do colo do útero são os de alto grau: 16, 18, 31, 33, 35, 39, 45, $46,51,52,56,58,59$ e 68 (NUNES JDC, et al., 2013). Os tipos 16 e 18 são responsáveis por $70 \%$ dos casos (NUNES PL, et al., 2020). 
O CCU surge devido à replicação desordenada do epitélio de revestimento do órgão, envolve o tecido subjacente (estroma) e pode acometer estruturas e órgãos próximos ou à distância. Os tipos de carcinomas invasores do colo do útero são: o epidermóide (mais incidente - $90 \%$ dos casos); e o adenocarcinoma (mais raro - representa 10\%) (INCA, 2020).

A neoplasia cervical é precedida por uma longa fase de doença pré-invasiva, denominada de lesão intraepitelial cervical (NIC), também conhecidas como displasias cervicais. Essas displasias são classificadas em: NIC1 (displasia leve confinada a um terço basal do epitélio), NIC2 (displasia moderada confinada a dois terços basal do epitélio) e NIC3 (displasia grave que se estende por mais de dois terços do epitélio ou carcinoma in situ (CIS) (JUNG S, et al., 2015). Esta é subdividida em graus baixo grau e alto grau, dependendo da proporção da espessura do epitélio que apresenta células maduras e diferenciadas (GIRIANELLI V, et al., 2014).

Assim, identificando as causas e os efeitos da dor na qualidade de vida das pacientes, trataríamos de forma efetiva a queixa álgica. Portanto, esse estudo visa avaliar a intensidade da dor nas pacientes com câncer cervical, por meio de escalas padronizadas e validadas para a língua portuguesa.

\section{MÉTODOS}

Estudo quantitativo, descritivo, do tipo exploratório, de corte transversal, realizado no ambulatório do setor de Oncologia do Hospital de Urgência de Sergipe, no município de Aracaju, no período de 2010 a 2014. Foi redigido um Termo de Consentimento Livre e Esclarecido, conforme as normas do Conselho Nacional de Saúde do Ministério da Saúde explicitadas na resolução 466/12.

A população da pesquisa foi de 53 pacientes diagnosticadas e tratadas com câncer de colo de útero no período de 2010 a 2014, de acordo com os prontuários disponibilizados no Hospital de Urgência de Sergipe (HUSE). Foram incluídas no estudo: mulheres com diagnóstico de câncer de colo do útero confirmado com anatomopatológico; àquelas que aceitaram participar da pesquisa após ter lido e assinado o Termo de Consentimento Livre e Esclarecido; àquelas que completaram o tratamento do câncer de colo do útero há pelo menos um ano; àquelas em vigência de tratamento quimioterápico ou radioterápico para câncer; àquelas que tiveram recidiva da doença; àquelas com idade maior que dezoito anos. Foram excluídas, pacientes que se recusaram a participar da pesquisa.

A coleta de dados se fez através de entrevistas individuais, a fim de evitar o não entendimento das perguntas dos questionários. Ambos foram estruturados com perguntas fechadas, já validadas. $O$ primeiro questionário aborda a escala visual analógica de avaliação da dor (EVA), que consiste numa escala de zero a dez sendo zero "nenhuma dor" e dez a "dor máxima imaginável", em que pacientes com dor leve apresentam valores de 1-3, moderada 4-6 e intensa 7-10 (NASCIMENTO JC, 2017).

O segundo consiste no questionário de McGill, que permite a avaliação quantitativa e qualitativa da dor, quanto ao seu aspecto sensorial, afetivo e avaliativo. Divide-se em 20 categorias, nas quais as de 1 a 10 representam as respostas sensitivas, as de 11 a 15 são de caráter afetivo, a 16 é de caráter avaliativo e as de 17 a 20 mesclam as outras categorias; depois de aplicado o questionário, somam-se as palavras (descritores) citadas pelo paciente para relatar a sua dor (máximo de 20) e também se somam os valores de intensidade de cada descritor (0-5), tendo somatória máxima de 78 (RUELA L, et al., 2017). As pacientes que apresentaram EVA "0" não fizeram parte da aplicação do questionário McGill (reduzindo o total de 53 pacientes para 34).

Os dados das escalas foram digitados em uma planilha do Excel e, posteriormente, compilados. A análise estatística foi realizada com o auxílio do software Statistical Package for the Social Sciences (SPSS), versão 20 (SPSS Inc., Chicago, IL, EUA). As variáveis contínuas foram descritas em média e desvio padrão, e as variáveis categóricas foram sumarizadas por meio de frequências simples e relativas.

Este estudo foi submetido ao Comitê de Ética em Pesquisa (CEP) da Universidade Tiradentes (UNIT), em conformidade com a Resolução 466/12 do Conselho Nacional de Saúde, tendo sido aprovado pelo parecer no 989.186 de $26 / 03 / 2015$. 


\section{RESULTADOS}

A queixa álgica foi um fenômeno relatado por 34 pacientes (64,1\%), seja no diagnóstico, ou no tratamento, ou na reabilitação ou em mais de uma dessas etapas. Quanto à avaliação da intensidade da dor pela Escala Visual Analógica (EVA), 22 entrevistadas (41,5\%) apresentaram dor intensa e 19 (35,8\%) não apresentaram dor. A dor leve foi relatada por 8 pacientes (15,2\%) e a dor moderada por 4 pacientes $(7,5 \%)$.

Ao analisar os descritores da dor, observou-se que dentre as 34 pacientes que referiam a queixa álgica, 0 parâmetro relativo à avaliação sensorial, à afetiva, à miscelânea e à avaliativa resultou numa média: 7,06 \pm $2,34,3,85 \pm 1,44,2,29 \pm 1,17$ e $0,97 \pm 0,17$, respectivamente. O descritor sensitivo mais utilizado pelas pacientes portadoras de câncer de colo uterino foi dor fina com $10,6 \%$, seguida pela dor em cólica com $9,7 \%$ desses dados (Tabela 1).

Tabela 1 - Qualidade sensitiva da dor.

\begin{tabular}{cc}
\hline Descritores & $\mathbf{n}(\%)$ \\
\hline Fina & $25(10,6)$ \\
Cólica & $23(9,7)$ \\
Calor & $15(6,4)$ \\
Agulhada & $13(5,5)$ \\
Latejante & $11(4,7)$ \\
Sensível & $11(4,7)$ \\
Pontada & $11(4,7)$ \\
Torção & $10(4,2)$ \\
Formigamento & $9(3,8)$ \\
Pesada & $9(3,8)$ \\
Puxão & $7(3,0)$ \\
Fisgada & $7(3,0)$ \\
Queimação & $7(3,0)$ \\
Ardor & $7(3,0)$ \\
\hline Total & $236(100)$
\end{tabular}

Fonte: Lima DC, et al., 2021; dados extraídos do Hospital de Urgência de Sergipe (HUSE).

O caráter afetivo demonstrou uma predominância quanto à dor enjoada com 22,9\% dessa categoria e uma menor taxa referente à dor mortal com $0,8 \%$ (Tabela 2).

Tabela 2 - Qualidade afetiva da dor.

\begin{tabular}{cc}
\hline Descritores & $\mathbf{n}(\%)$ \\
\hline Enjoada & $30(22,9)$ \\
Cansativa & $22(16,8)$ \\
Castigante & $13(9,9)$ \\
Miserável & $13(9,9)$ \\
Amedrontada & $10(7,6)$ \\
Enlouquecedora & $9(6,9)$ \\
Atormentada & $7(5,3)$ \\
Exaustiva & $6(4,6)$ \\
Cruel & $6(4,6)$ \\
Maldita & $6(4,6)$ \\
Apavorante & $4(3,0)$ \\
Sufocante & $2(1,5)$ \\
Aterrorizante & $2(1,5)$ \\
Mortal & $1(0,8)$ \\
\hline Total & $131(100)$
\end{tabular}

Fonte: Lima DC, et al., 2020; dados extraídos do Hospital de Urgência de Sergipe (HUSE). 
A análise demonstrou que quanto a qualidade avaliativa da dor, as entrevistadas demonstraram que a dor é melhor descrita como chata $(33,3 \%)$ e que incomoda $(33,3 \%)$, em segundo lugar a dor insuportável foi a mais relatada $(37,2 \%)$ (Tabela 3$)$.

Tabela 3 - Qualidade avaliativa da dor.

\begin{tabular}{cc}
\hline Descritores & $\mathbf{n ~ ( \% )}$ \\
\hline Chata & $11(33,3)$ \\
Que incomoda & $11(33,3)$ \\
Insuportável & $9(27,2)$ \\
Forte & $2(6,1)$ \\
\hline Total & $33(100)$
\end{tabular}

Fonte: Lima DC, et al., 2021; dados extraídos do Hospital de Urgência de Sergipe (HUSE).

Os resultados referentes aos descritores da miscelânea demonstraram maior prevalência da dor aborrecida (15,4\%), seguida pela dor fria (12,8\%) (Tabela 4).

Tabela 4 - Qualidade de miscelânea da dor.

\begin{tabular}{cc}
\hline Descritores & $\mathbf{n}(\%)$ \\
\hline Aborrecida & $12(15,4)$ \\
Fria & $10(12,8)$ \\
Espalha & $7(9,0)$ \\
Penetra & $6(7,7)$ \\
Repuxa & $5(6,4)$ \\
Espreme & $5(6,4)$ \\
Torturante & $5(6,4)$ \\
Irradia & $4(5,1)$ \\
Aperta & $4(5,1)$ \\
Gelada & $4(5,1)$ \\
Dá náusea & $4(5,1)$ \\
Rasga & $3(3,8)$ \\
Agonizante & $3(3,8)$ \\
Pavorosa & $2(2,6)$ \\
Atravessa & $2(2,6)$ \\
Adormece & $2(2,6)$ \\
\hline Total & $236(100)$
\end{tabular}

Fonte: Lima DC, et al., 2021; dados extraídos do Hospital de Urgência de Sergipe (HUSE).

Quanto aos resultados relativos ao índice de avaliação da dor (IAD), notou-se uma predominância do caráter sensorial com média de 16,68 $\pm 7,34$, seguido pelo caráter afetivo com $5,79 \pm 3,02$, pelo caráter miscelânea com $5,26 \pm 3,54$ e pelo caráter avaliativo com $2,53 \pm 1,69$.

\section{DISCUSSÃO}

Uma estimativa mundial aponta que ocorreram 18 milhões de pessoas diagnosticadas com algum tipo de câncer e a dor associada com essa doença causa séria preocupação (BRAY $F$, et al; 2018). Esse sintoma é relatado em aproximadamente $50 \%$ das pessoas com doença e em até $90 \% \mathrm{com}$ a neoplasia na fase avançada (OMS, 2017).

A dor é frequentemente citada como o mais crítico, interferindo profundamente nas atividades da vida diária do paciente, juntamente com a sua adesão ao tratamento. A prevalência de dor no câncer é estimada entre $25 \%$ a $50 \%$ dos pacientes recém-diagnosticados, $33 \%$ e $80 \%$ dos submetidos a tratamento ativo, e superior a $75 \%$ para aqueles com doença avançada (MENDES CM, et al., 2020).

O aparecimento de dor no carcinoma cervical ocorre tardiamente, o que leva a uma procura por médico pelas mulheres somente quando a doença já está avançada, diminuindo as chances de cura e de um tratamento menos invasivo (LOPES V e RIBEIRO J, 2019). 
Nesse estudo, 34 pacientes $(64,1 \%)$ relataram dor, seja no diagnóstico, no tratamento, na reabilitação ou em mais de uma dessas etapas. Já na avaliação da intensidade da dor pela EVA, 22 entrevistadas $(41,5 \%)$ referiram dor intensa e 19 (35,8\%) não apresentaram dor.

No estudo de Te Boveldt N, et al. (2013), a maioria dos pacientes oncológicos referiram ausência de dor. Costa A e Chaves M (2012), assim como Pimenta CA, et al. (2000) encontraram nas suas análises, uma dor oncológica de moderada intensidade. Silva e Gradin (2010) verificaram que $50 \%$ das pacientes com câncer de mama tinham dor descrita como leve (NGAMKHAM S, et al; 2012). Silva et al. (2011) encontrou resultado semelhante ao nosso, no qual a maioria das mulheres com câncer cervical tinha uma dor intensa. Deste modo, os estudos divergem quanto à intensidade da dor associada ao câncer, fato que pode ser explicado, talvez, pela variabilidade de órgãos acometidos.

Dentre as 34 pacientes que referiram queixa álgica nessa pesquisa, os descritores sensoriais foram os mais mencionados (com média 7,06 $\pm 2,34$ ), o que coincidiu com os estudos de Silva V e Gradim C (2010), Costa A e Chaves M (2012), Ngamkham S, et al. (2012). Não se sabe ao certo se a dor oncológica apresenta maior associação com os descritores sensoriais, ou se essa associação ocorre somente pela distribuição desproporcional desses descritores (42 de um total de 78) (RETT M, et al., 2012). Acredita-se que a pessoa avaliada seja induzida a escolher mais descritores sensoriais.

O descritor sensitivo mais utilizado pelas pacientes foi dor fina com 10,6\%, assim como nos estudos de Costa A e Chaves M (2012), Costa A (2014). Silva V e Gradim C (2010) constataram que a maioria dos entrevistados descreveu a dor no caráter sensitivo como fisgada e ferroada; já Silva T, et al. (2011), observou predominância de uma dor latejante, na categoria sensitiva. Portanto, nota-se que os resultados são distoantes quanto o descritor sensorial mais mencionado pelos pacientes oncológicos. Essa discordância pode ser consequência da dificuldade dos entrevistados em escolher os itens qualitativos da queixa dolorosa, por não compreenderem muito bem o seu significado, necessitando de habilidades cognitivas para uma escolha mais acurada.

No questionário de McGill, a dimensão sensorial-discriminativa é influenciada pelos sistemas espinais de condução rápida (dor aguda); já a dimensão afetiva-motivacional é influenciada pelos sistemas espinais de condução lenta (dor crônica) (SILVA A, et al., 2018). Observou-se então que a dor referida pelas pacientes com câncer de colo uterino era de caráter sensitivo, sendo assim tida como aguda.

A categoria sensorial descreve as propriedades mecânicas, térmicas, de vividez e espaciais da dor, sendo, portanto, o principal critério para a definição da dor neuropática; já a categoria afetiva descreve a dimensão afetiva da dor nos aspectos de tensão, medo, cansaço e respostas neurovegetativas (RETT M, et al., 2012; BOUREAU F, et al., 1990). Logo, nesse estudo, a dor do câncer cervical tem mais características neuropáticas.

Costa A e Chaves M (2012), Costa A (2014) constataram que o IAD era mais intenso na categoria sensitiva. Resultado semelhante foi verificado no nosso estudo, com predomínio também do caráter sensorial com uma média de 16,68 $\pm 7,34$. Fatores psicológicos interferem não apenas no caráter afetivo da dor, mas também no sensitivo, avaliativo e de miscelânea, superestimando assim o índice total da dor (NGAMKHAM S, et al., 2012).

O Questionário de Mcgill, então, permitiu a análise da dor quantitativamente e qualitativamente, uma vez que apenas a mensuração da algia pela Escala Visual Analógica não oferece parâmetros suficientes para uma avaliação fidedigna.

\section{CONCLUSÃO}

A partir desse estudo, concluiu-se que a dor intensa esteve presente na maioria das pacientes com a neoplasia de colo uterino em algum estágio da doença. Observou-se também que tanto o número de descritores, quanto o índice da dor tiveram a predominância de um caráter sensorial, necessitando mais estudos para comprovação da associação dessa categoria com a dor oncológica, sobretudo no câncer em questão. Vale ressaltar que uma das falhas do nosso estudo, foi a não associação da dor com o estadiamento e o tratamento da neoplasia. Por conseguinte, novos estudos devem ser realizados para verificar se a dor é fruto do próprio câncer, do seu estadiamento ou do seu tratamento. 


\section{REFERÊNCIAS}

1. ARAUJO L, ROMERO B. Dor: avaliação do 5o sinal vital. Uma reflexão teórica. Rev. dor, 2015; 16(4): $291-296$.

2. BICCA G, et al. Infecção por HPV e câncer cervical: uma revisão de triagem e estratégias preventivas nos países desenvolvidos e políticas brasileiras. DST - J bras Doenças Sex Transm, 2013; 25(3): 157.

3. BOUREAU F, et al. Study of verbal description in neuropathic pain. Pain, 1990; 41: 145-152.

4. BRASIL. Ministério da Saúde. Cuidados paliativos oncológicos: controle da dor. 2001.

5. BRAY F, et al. Global cancer statistics 2018: GLOBOCAN estimates of incidence and mortality worldwide for 36 cancers in 185 countries. CA: a cancer journal for clinicians, Hoboken, 2018; 68(6): 394-424.

6. CARVALHO K, et al. A relação entre HPV e câncer de Colo de Útero: Um panorama a partir da produção bibliográfica da área. Revista Saúde em Foco - Edição no 11 - Ano: 2019.

7. COSTA A. Dor e qualidade de vida: Caracterização e avaliação de pacientes oncológicos com feridas tumorais. Brasília. Tese [Mestrado em Enfermagem em Área Concentração] - Universidade de Brasília; 2014.

8. COSTA A, CHAVES M. Dor em pacientes oncológicos sob tratamento quimioterápico. Rev Dor. 2012; 13(1): 45-9.

9. FACINA T. Estimativa 2014 - Incidência de Câncer no Brasil. Revista Brasileira de Cancerologia. $2014 ; 60$.

10. GARCIA J. Dor neuropática. Sociedade Brasileira para Estudos da Dor, São Paulo, 2010; 2: 1-7.

11. GIRIANELLI V, et al. Os grandes contrastes da mortalidade por câncer de colo uterino e mama no Brasil. Rev Saúde Pública. 2014; 48(3): 459-467.

12. GRANER K. et al. Dor em oncologia: intervenções complementares e alternativas ao tratamento medicamentoso. Temas psicol, Ribeirão Preto, 2010; 18(2).

13. GREEN MC, et al. Câncer de colo uterino em idosas: revisão de literatura. Revista Eletrônica Acervo Saúde, 2020; 52: 3589.

14. INSTITUTO NACIONAL DE CÂNCER JOSÉ ALENCAR GOMES DA SILVA. Atlas On-line de Mortalidade [internet]. 2019. Brasília, DF: Ministério da Saúde. [acesso em 2021 mar 6]. Disponível em: https://mortalidade.inca.gov.br/MortalidadeWeb/

15. INSTITUTO NACIONAL DE CÂNCER JOSÉ ALENCAR GOMES DA SILVA. Estimativa 2020: incidência de câncer no Brasil / Instituto Nacional de Câncer José Alencar Gomes da Silva. - Rio de Janeiro: INCA, 2019.

16. JUNG S, et al. Progression of naive intraepithelial neoplasia genome to aggressive squamous cell carcinoma genome of uterine cervix. Oncotarget. 2015; 6: 4385-4393

17. LOPES V, RIBEIRO J. Fatores limitadores e facilitadores para o controle do câncer de colo de útero: uma revisão de literatura. Ciênc. Saúde coletiva, Rio de Janeiro, 2019; 24(9): 3431-3442.

18. MENDES CM, et al. Índice de dor neuropática em pacientes oncológicos e conduta farmacológica. FAG Journal of Health 4. 2020; 424-428.

19. NASCIMENTO JC. Avaliação da dor em paciente com câncer em cuidados paliativos a luz da literatura. Saúde \& ciência em ação - Revista Acadêmica do Instituto de Ciências da Saúde, 2017; 3: 01.

20. NGAMKHAM S, et al. The McGill Pain Questionnaire as a Multidimensional Measure in People with Cancer: An Integrative Review. Pain Manag Nurs. 2012; 13(1): 27-51.

21. NUNES J, et al. Identificação molecular do HPV em infecções do colo uterino no Brasil: revisão. FEMINA. 2013; 41(2): 93-98.

22. NUNES PL, et al. HPV e o desenvolvimento de Neoplasia do colo do Útero. Braz. J. Hea. Rev., Curitiba, 2020; 3(5): 14566-14569.

23. OLIVEIRA S, et al. A espiritualidade no enfrentamento da dor em pacientes oncológicos: revisão sistemática. BrJP, São Paulo, 2020; 3(2): 158-163.

24. Organização Mundial de Saúde - OMS. 2017. Disponível em: <http://www.who.int/eportuguese/countries/bra/pt/>. Acesso em: 6 mar. 2021.

25. PIMENTA CA, et al. Dor crônica e depressão: estudo em 92 doentes. Rev.Esc.Enf.USP. 2000; 34(1): 76-83.

26. PIMENTEL A, et al. A percepção da vulnerabilidade entre mulheres com diagnóstico. Texto Contexto Enferm. 2011; 20(2): 255-62.

27. RETT $M$, et al. A cinesioterapia reduz a dor no membro superior de mulheres submetidas à mastectomia ou quadrantectomia. Rev. Dor, 2012; 13: 3.

28. RUELA L, et al. Avaliação da dor em pacientes em tratamento quimioterápico: utilização do questionário McGill. Rev. dor, São Paulo, 2017; 18(2): 156-160.

29. SCHMIDT B. The Neurobiology of Cancer Pain. Neuroscientist. 2014; 20(5): 546-562.

30. SILVA A, et al. Caracterização da dor decorrente de traumas perineais em mulheres com parto vaginal. BrJP, São Paulo, 2018; 1(2): 158-162.

31. SILVA T, et al. Avaliação da dor em pacientes oncológicos. Rev. enferm. UERJ. 2011; 19(3): p. 359-63

32. SILVA V, GRADIM C. Avaliação da dor em mulheres com câncer de mama submetidas à exérese da rede linfática axilar. Cogitare Enferm. 2010; 15(4): 646-51.

33. SOUSA AC, et al. Caracterização das alterações citopatológicas e fatores de riscos associados ao desenvolvimento do câncer de colo uterino. Revista Uningá Review, [S.I.], 2017; 30: 1.

34. TE BOVELDT N, et al. Pain and Its Interference with Daily Activities in Medical Oncology Outpatients. Pain Physician. 2013; 16: 379-389.

35. THANTHONG S, et al. Comparison of efficacy of meperidine and fentanyl in terms of pain management and quality of life in patients with cervical cancer receiving intracavitary brachytherapy: a double-blind, randomized controlled trial. Support Care Cancer. 2017; 25(8): 2531-2537.

36. THOMAZ A. Dor oncológica: conceitualização e tratamento farmacológico. Onco\&. 2010; 24-29. 